\title{
A dual fluorescent reporter for the investigation of methionine mistranslation in live cells
}

\author{
ANA CRISTINA GOMES, ${ }^{1}$ ANNA J. KORDALA, ${ }^{1}$ RITA STRACK, ${ }^{1}$ XIAOYUN WANG, ${ }^{1}$ RENAUD GESLAIN, ${ }^{1,3}$ \\ KAMILA DELANEY, ${ }^{1}$ WESLEY C. CLARK, ${ }^{1}$ ROBERT KEENAN, ${ }^{1}$ and TAO PAN ${ }^{\mathbf{1}, 2}$ \\ ${ }^{1}$ Department of Biochemistry and Molecular Biology, University of Chicago, Chicago, Illinois 60637, USA \\ ${ }^{2}$ Institute of Biophysical Dynamics, University of Chicago, Chicago, Illinois 60637, USA
}

\begin{abstract}
In mammalian cells under oxidative stress, the methionyl-tRNA synthetase (MetRS) misacylates noncognate tRNAs at frequencies as high as $\mathbf{1 0} \%$ distributed among up to $\mathbf{2 8}$ tRNA species. Instead of being detrimental for the cell, misincorporation of methionine residues in the proteome reduces the risk of oxidative damage to proteins, which aids the oxidative stress response. tRNA microarrays have been essential for the detection of the full pattern of misacylated tRNAs, but have limited capacity to investigate the misacylation and mistranslation mechanisms in live cells. Here we develop a dual-fluorescence reporter to specifically measure methionine misincorporation at glutamic acid codons GAA and GAG via tRNA ${ }^{\text {Glu }}$ mismethionylation in human cells. Our method relies on mutating a specific Met codon in the active site of the fluorescent protein mCherry to a Glu codon that renders mCherry nonfluorescent when translation follows the genetic code. Mistranslation utilizing

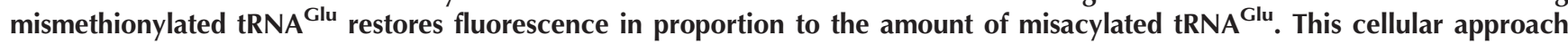
works well for both transient transfection and established stable HEK293 lines. It is rapid, straightforward, and well suited for high-throughput activity analysis under a wide range of physiological conditions. As a proof of concept, we apply this method to characterize the effect of human tRNA ${ }^{\text {Glu }}$ isodecoders on mistranslation and discuss the implications of our findings.
\end{abstract}

Keywords: FACS; mistranslation; fluorescent protein; reporter; tRNA isodecoder

\section{INTRODUCTION}

Protein expression represents a huge investment in cellular resources. Organisms developed mechanisms for reduction of error frequencies and also tolerance strategies that allow them to cope with the physiological consequences of protein synthesis errors. During translation, aminoacyl-tRNA synthetases (AARSs) play a crucial role in maintaining high accuracy (Schimmel and Soll 1979; Carter 1993). Each amino acid is specifically recognized out of 20 by its cognate AARS and esterified to the appropriate tRNA to form aminoacyl-tRNA. The experimentally determined error rate in amino acid selection in vitro is commonly in the range of $10^{-4}$ to $10^{-5}$ (Ibba and Soll 2000; Sankaranarayanan and Moras 2001). These values are explained by kinetic discrimination mechanisms and proofreading (or editing) occurring in a distinct catalytic site of many AARSs. This ensures that the correctly charged cognate tRNA is inserted into the A-site in the ribosome, contributing to the overall protein synthesis fidelity before peptide bond formation.

\footnotetext{
${ }^{3}$ Present address: College of Charleston, Charleston, SC 29424, USA Corresponding author: taopan@uchicago.edu

Article published online ahead of print. Article and publication date are at http://www.rnajournal.org/cgi/doi/10.1261/rna.054163.115.
}

As recently demonstrated, however, high levels of tRNA misacylation with methionine mediated by methionyltRNA synthetase (MetRS) can occur in vivo in eukaryotes (Netzer et al. 2009; Wiltrout et al. 2012; Lee et al. 2014). In mammalian cells, tRNA mismethionylation occurs at a basal level of $\sim 1 \%$ distributed among up to eight tRNA species and can increase up to $10 \%$ distributed among up to 28 tRNA species without any known detrimental effects for the cell. Elevated tRNA mismethionylation is triggered by reactive oxygen species (ROS), and is part of a regulated defense mechanism against oxidative stress (Netzer et al. 2009; Lee et al. 2014).

Cells invest energy and resources for both scavenging ROS and reversing oxidative damage (Imlay 2008; Avery 2011). Methionine at the surface or near the active site of proteins can scavenge ROS through its reactive sulfur group, which is immediately converted back to its reduced form by the methionine sulfoxide reductases system. Oxidative stress decreases the specificity of MetRS and increases

\footnotetext{
(C) 2016 Gomes et al. This article is distributed exclusively by the RNA Society for the first 12 months after the full-issue publication date (see http://rnajournal.cshlp.org/site/misc/terms.xhtml). After 12 months, it is available under a Creative Commons License (Attribution-NonCommercial 4.0 International), as described at http://creativecommons.org/licenses/by$\mathrm{nc} / 4.0 /$.
} 
mismethionylation for a range of noncognate tRNAs. In mammalian cells, the activity of MetRS can be modulated swiftly by posttranslational modification and has been shown to control several cellular responses such as amino acid limitation (Kwon et al. 2011). An increase in ROS results in specific Ser209 and Ser825 dual phosphorylation by the extracellular signal-related kinase (ERK1/2), which is responsible for the decrease in MetRS fidelity (Lee et al. 2014). The resulting misacylated tRNAs are used in translation (Netzer et al. 2009; Lee et al. 2014). Misincorporation of methionine residues reduces the risk of protein damage due to an increased capacity to capture and inactivate ROS (Luo and Levine 2009).

To discover and characterize tRNA misacylation, we applied a method involving pulse radiolabeling of cells with $\left[{ }^{35} \mathrm{~S}\right]-$ Met, followed by lysis and total RNA hybridization on tRNA microarrays which allowed the identification of the full pattern of mismethionylated tRNAs (Netzer et al. 2009; Jones et al. 2011; Wiltrout et al. 2012). The tRNA microarray contains complementary oligonucleotide spots corresponding to all tRNAs for a given organism, so that all tRNAs can be assayed in a single experiment. Not all tRNAs are equally mismethionylated. Although tRNA mismethionylation is present both in vivo and in vitro using purified MetRS, more tRNA species are mismethionylated in vivo than in vitro, suggesting that MetRS alone is necessary but not sufficient to produce the in vivo pattern.

An additional unknown factor of tRNA misacylation is the effect of tRNA isodecoders - tRNAs with the same anticodon but different body sequences. tRNA isodecoder genes are particularly prominent in the mammalian genome (Goodenbour and Pan 2006). For example, the tRNA ${ }^{\text {Glu }}$ family in the human genome consists of seven isodecoders, two with the CTC anticodon and five with the TTC anticodon. Because many AARSs recognize a distinct set of tRNA body sequences, it is possible that each isodecoder may have a distinct ability to be misacylated in cells. Hence, the expression level of these isodecoders in a specific cell line or tissue type may influence the mismethionylation level of each tRNA family.

tRNA microarrays cannot be readily applied to address these questions. Its resolution is not sufficient to distinguish individual members of a tRNA isodecoder family (Dittmar et al. 2004, 2006). Furthermore, tRNA microarrays are limited to measuring tRNA mismethionylation from total RNA extracts and hence cannot follow mistranslation dynamics in live cells.

Here we report the development of an in vivo reporter system that enables the investigation of Met mistranslation in live mammalian cells. Our method uses a dual-fluorescent protein strategy coupled with flow cytometry. The dual fluorescent protein is an EGFP-mCherry fusion where the EGFP is always the wild type (WT) and therefore reports the expression level of the protein. The mCherry contains a single codon mutation of Met72 (ATG) to a non-Met codon such as Glu (GAA or GAG). Met72 is one of the three amino acid residues in mCherry that undergoes an auto-catalyzed covalent reaction to form the fluorophore; thus, the reporter should only acquire fluorescence upon misincorporation of Met at position 72. We show that this reporter works well both in transiently transfected and in stably transfected cells. Using this system, we further examine the effect of transfecting individual tRNA ${ }^{\text {Glu }}$ isodecoders on mistranslation. Our results demonstrate a robust system capable of studying the cellular mechanisms of tRNA misacylation and mistranslation under a wide range of physiological conditions.

\section{RESULTS AND DISCUSSION}

\section{Dual fluorescence reporters to quantify Met mistranslation in live cells}

Although the development of tRNA microarrays led to the discovery of tRNA misacylation triggered by oxidative stress, for a better understanding of the mechanisms involved, the study of live cells will be essential. The surge of fluorescent protein reporters revolutionized biological studies. Quantification of multicolor fluorescence emission in specific cell populations can be performed at high precision and speed by flow cytometry. mCherry was developed by directed mutagenesis of mRFP1, a monomeric mutant of DsRed (Campbell et al. 2002). The result is a fast maturating protein, highly photostable, and resistant to photobleaching (Shaner et al. 2004). The Met72 residue is crucial for mCherry fluorescence, being one of the three amino acid residues forming the fluorophore (Shaner et al. 2004). Initial experiments using the single fluorescent protein expressed in E. coli showed that when Met72 (ATG) is mutated to Lys (AAA, AAG), Asp (GAC, GAT), or Glu (GAG, GAA), its fluorescence was completely lost, even though the expressed mutant proteins were stable in E. coli (Fig. 1A; Supplemental Fig. S1). This "all-or-nothing" effect of substitutions at this specific position represents a unique resource to study the translation accuracy.

When using only a single fluorescent reporter protein, a large ambiguity might occur due to intrinsic variations in protein expression. We bypassed this problem by developing a dual-fluorescence reporter consisting of an in-frame fusion of EGFP and mCherry expressed under the control of the CMV promoter and SV40 terminator for mammalian cells. The excitation and emission spectra of GFP and mCherry have minimal overlap, allowing specific detection of their distinct signals in the same sample (Fig. 1B; Supplemental Fig. S2). GFP acts as an internal normalization control for quantification of mCherry fluorescence, accounting for the level of mRNA abundance and efficiency of translation initiation. Increasing amounts of WT EGFP-mCherry plasmid were transiently transfected into HeLa cells, resulting in proportionally high fluorescent values (Fig. 1B). The expression of EGFP and WT mCherry as a translational fusion did 
A
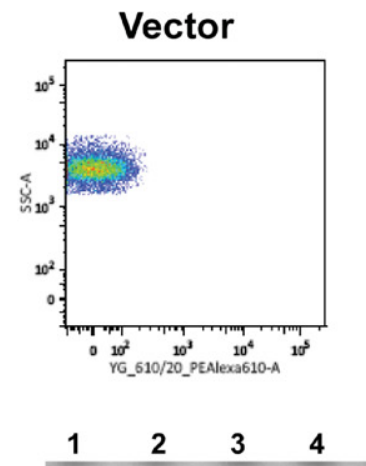

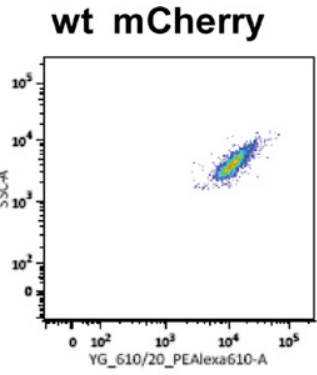

$\begin{array}{llll}5 & 6 & 7 & 8\end{array}$

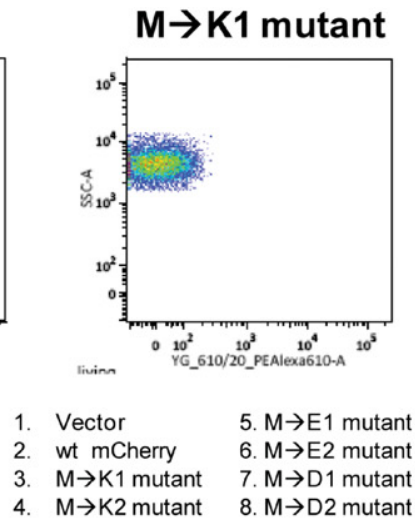

C

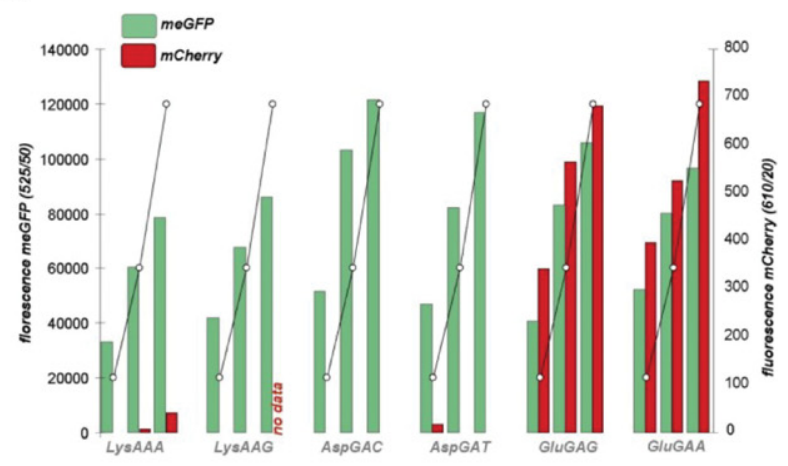

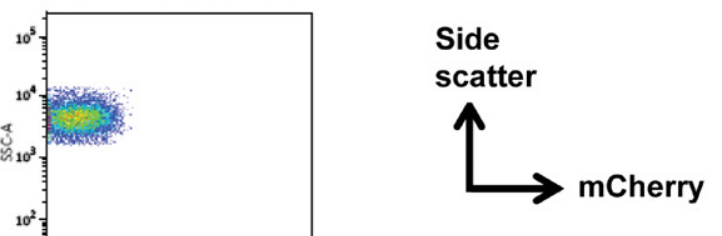

$M \rightarrow K 1$ mutant
B

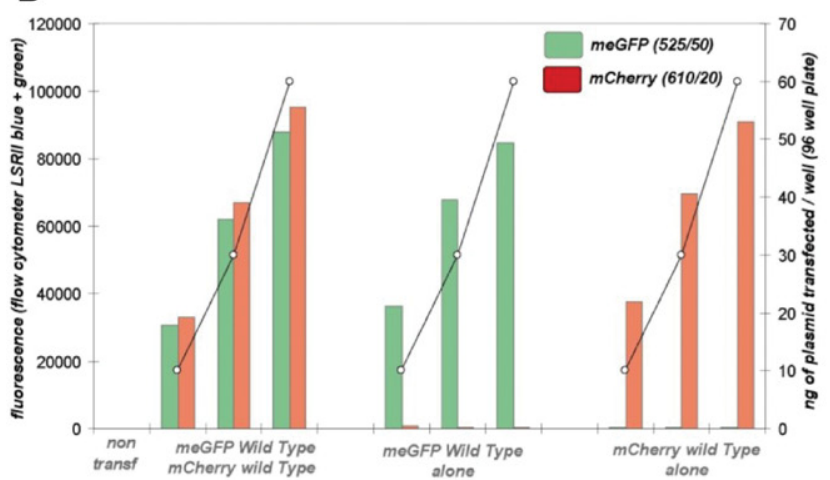

FIGURE 1. mCherry fluorescent reporter for mistranslation. One of the three amino acid residues that form the fluorophore in $\mathrm{mCherry}$ is Met72. (A) mCherry lost fluorescence in E. coli when Met72 was mutated to Lys (AAA, AAG), Asp (GAC, GAT), and Glu (GAG, GAA), as determined by flow cytometry (AAG mutant shown). All mutant proteins in E. coli are as stable as the WT protein as shown by Coomassie brilliant blue stain of the total cell protein (bottom). (B) EGFP and WT mCherry transiently transfected to HeLa cells. Transfection was done either using the fusion protein (left) or just the EGFP or mCherry alone, showing little signal overlap between these two proteins. $Y$-axis on the left (bar graphs) shows EGFP and mCherry at the same intensity; $y$-axis on the right (circles) shows the amount of plasmids used in transfection. $(C)$ EGFP-mCherry dual fluorescence reporter mutants were transiently transfected to HeLa cells and cells were analyzed by flow cytometry. Cells transfected with the reporter harboring a Glu mutation show high residual mCherry fluorescence. Same amounts of plasmids as in panel $B$ were used in transfection. $Y$-axis on the left (green bars) shows eGFP intensity; $y$-axis on the right (red bars) shows mCherry intensity. Note that the left and right $y$-axes have different scales.

not affect their fluorescence, substantiating the robustness of this dual-fluorescence reporter. Dual reporters harboring mCherry codon mutations for Lys, Asp, or Glu at position 72 revealed distinct fluorescent features in HeLa cells (Fig. 1C). Lys and Asp at position 72 ablated mCherry fluorescence, whereas Glu72 mutants (GAA and GAG) showed residual mCherry fluorescence, $\sim 0.5 \%$ of the WT mCherry.

Lys, Asp, and Glu codon substitutions were chosen based on our previously published results identifying their cognate tRNAs as misacylation targets in human cells (Netzer et al. 2009). For the Glu72 codon mutation, both the appearance and the magnitude of mCherry fluorescence match the tRNA misacylation results detected by microarrays (Netzer et al. 2009). To our surprise, the Lys and Asp codon mutations at this specific location in mCherry did not match the tRNA misacylation result revealed by microarrays, even though Lys-to-Met or Asp-to-Met substitutions in specific cellular proteins have already been identified in the cellular proteome by mass spectrometry (Netzer et al. 2009; Lee et al. 2014). This result may be due to elongation factor and/or ribosome selection of misacylated tRNAs in a codon-context-dependent manner (Wolfson et al. 2001; Olejniczak and Uhlenbeck 2006). This tRNA selection on the ribosome may also explain why an ATG-to-AAG (Metto-Lys) in a different fluorescent reporter, TagRFP, did show fluorescence in a mismethionylation-dependent fashion (Lee et al. 2014). An alternative explanation for our results is that cells may hyperselect mismethionylated tRNA ${ }^{\text {Glu }}$ in a codon-context-dependent manner. In any case, how such selections occur in mammalian cells requires further investigation.

One concern for our result is that the mCherry fluorescence in the Glu72 mutants might be derived from intrinsically weak fluorescence of a Glu72-containing fluorophore. This is unlikely as the Glu72 mutant mCherry protein expressed and purified from E. coli is not fluorescent, similar to the purified Asp72 and Lys72 mutant proteins (Supplemental Fig. S1). As an additional validation, we isolated the 
GFP-mCherry fusion protein from HEK293T using GFP antibody, digested the protein with Glu-C protease that specifically cleaves proteins at Glu residues, and examined the digestion products by MALDI (Supplemental Fig. S3). Chromophore formation in the WT mCherry requires chemical transformation of three residues including Met72. The same chemistry involving Glu72 would eliminate the ability of Glu-C to cleave the Glu72-mCherry protein at this position. Compared to the WT mCherry, we found two new peptides with the appropriate molecular mass derived from Glu-C cleavage at Glu72, suggesting that the Glu72-mCherry protein does not readily form a fluorophore.

We set out to provide additional evidence that the fluorescence in the Glu72 mutants (GAA and GAG) can be used as a measure of tRNA mismethionylation. However, before further studies we performed the stable integration of the dual fluorescence reporter into the genome of HEK293 cells. Stable lines are needed to ensure convenient and long-term studies under a wide range of physiological conditions. The stable line strategy is described in Figure 2A. Based on the transient transfection results shown in Figure 1, we mutated the ATG WT codon for Met72 to AAG (Lys), GAC (Asp), GAA, and GAG (Glu). The mCherry fluorescence of the mutants was quantified by flow cytometry by the size of the $\mathrm{EGFP}+/ \mathrm{mCherry}+$ cell population relatively to WT (Fig. $2 \mathrm{~B}, \mathrm{C})$. As we have seen in HeLa cells, the stable GAA and GAG cell lines showed substantial mCherry fluorescence. For these two single clones, $16.7 \%$ of GAA and $33.6 \%$ of the GAG cells are positive for mCherry. Consistent with the flow cytometry results, mCherry fluorescence in the Glu72 construct cells can be readily seen using fluorescence microscopy (Supplemental Fig. S4).
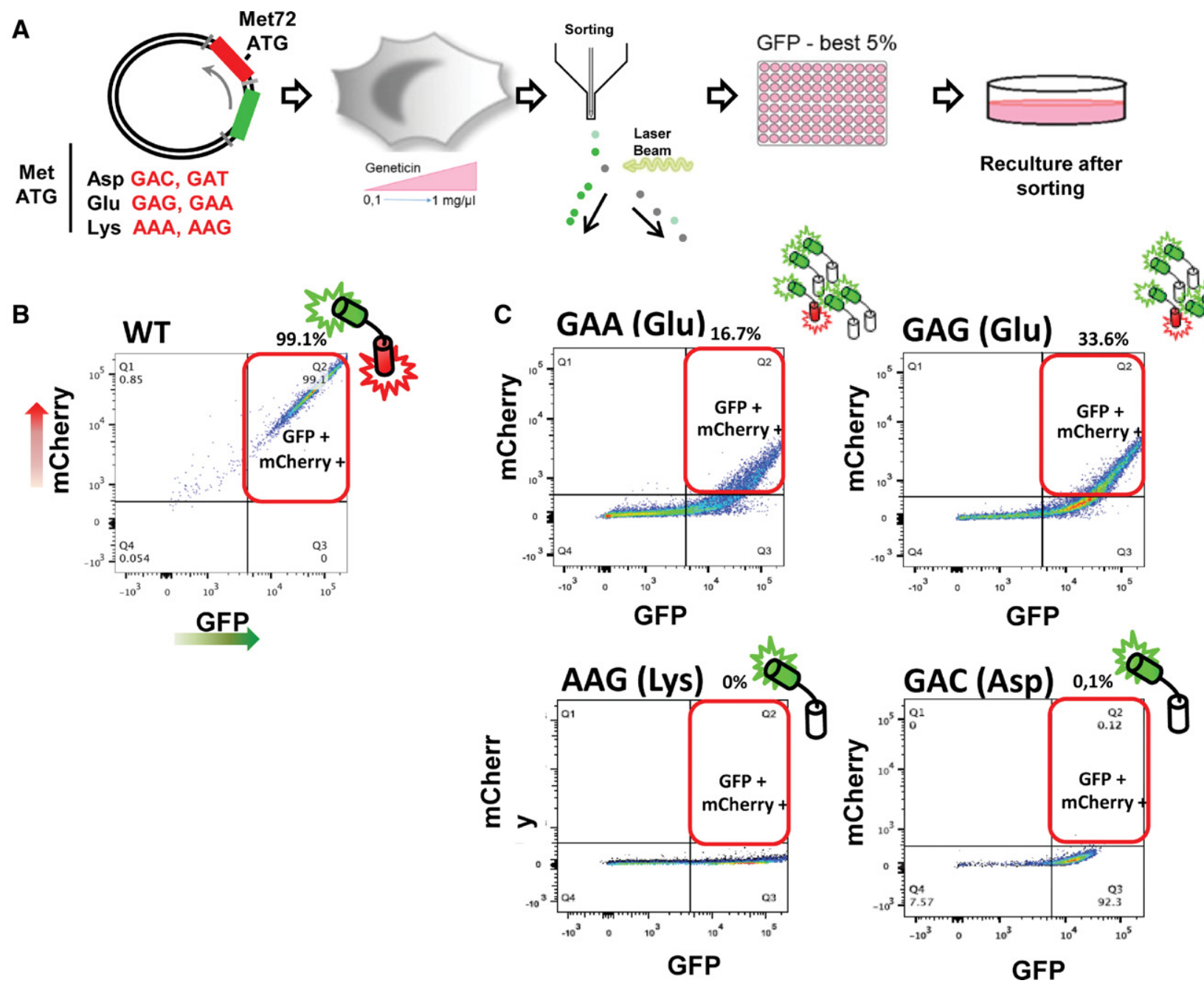

FIGURE 2. Experimental setup to quantify Met mistranslation in live cells using dual fluorescence reporters. (A) Schematic description of strategy used for creating the stable cell lines. (B) FACS plot for HEK293 cells stably transfected with the WT plasmid. (C) FACS plot of the cells stably transfected with GAA (Glu), GAG (Glu), AAG (Lys), and GAC (Asp) mutant plasmid. The quadrants in red correspond to the strongly GFP+/mCherry+ cell population. The percentage of cells in this population is also indicated. Quadrant gates were drawn based on fluorescence emission of the WT (positive control) cell population. Debris and doublets were gated out of the analysis. 
Recovery of mCherry fluorescence can occur due to the translation of the mutated codon by mismethionylated $\mathrm{tRNA}^{\mathrm{Glu}}$, restoring the Glu residue to the original Met. To confirm this, we generated oxidative stress by treating cells with arsenite which is known to induce tRNA mismethionylation to high levels (Fig. 3A; Netzer et al. 2009). To rule out that different maturation times of GFP and mCherry may introduce artifacts in quantitation, we examined the slope of the GFP/mCherry plots of the WT construct cells with and without arsenite treatment (Supplemental Fig. S5). The slopes were identical, indicating that both proteins do not have significantly different maturation times under these conditions.

Because mismethionylated $\mathrm{tRNA}^{\mathrm{Glu}}$ is also present in untreated cells, the specific increase of mismethionylated $\mathrm{tRNA}^{\text {Glu }}$ is only up to 1.5- to twofold. In GAA cells treated with arsenite, the EGFP+/mCherry+ population increased from $16.7 \%( \pm 4.5)$ of the total number of viable cells to $24.6 \%( \pm 5.6)$ after $12 \mathrm{~h}(1.5 \times)$ and up to $29.7 \%( \pm 3.3)$ after $24 \mathrm{~h}(1.8 \times$; Fig. $3 \mathrm{~B})$. In GAG cells, this increase was from $33.6 \%( \pm 8.2)$ to $47.9 \%( \pm 6.1)$ after $12 \mathrm{~h}(1.4 \times)$ and to up to $48.2 \%( \pm 1.5)$ after $24 \mathrm{~h}$ of arsenite treatment $(1.4 \times$; Fig. 3C). As a control, the AAG cells did not show additional mCherry fluorescence after arsenite treatment (Fig. 3D). Remarkably, the number of cells with high fluorescence (ar- bitrarily set as >1000 a.u. in this work) increased up to 8.8fold for the GAA cells $(2.3 \%$ to $20.3 \%)$ and up to 2.8 -fold for the GAG cells (10.5\% to 29\%) upon arsenite treatment (Supplemental Fig. S6). This result indicates that, combined with flow cytometry, these cell lines possess high sensitivity for physiological studies or chemical library screening for small molecule inhibitors of mistranslation.

We performed one more experiment to show that the arsenite-induced change in mistranslation is indeed dependent on ERK1/2 activity as previously described (Lee et al. 2014). Both GAA and GAG stable lines were treated with arsenite and FR180204, a selective inhibitor of the ERK1/2 activity that acts by competing with ATP binding (Ohori et al. 2005). ERK1/2 can be activated by ROS and phosphorylate MetRS at two specific residues, Ser209 and Ser825. Through this mechanism, ERK1/2 is responsible for a decrease in aminoacylation accuracy of MetRS (Lee et al. 2014). Indeed, in cells treated simultaneously with FR180204 and arsenite, there was no significant change in the percentage of cells with mCherry fluorescence (Fig. 4). However, the presence of FR180204 did not eliminate the mCherry fluorescence without arsenite, similar to and consistent with the previous observation in TagRFP that the basal tRNA mismethionylation without stress is not related to ERK1/2 phosphorylation of MetRS (Lee et al. 2014).

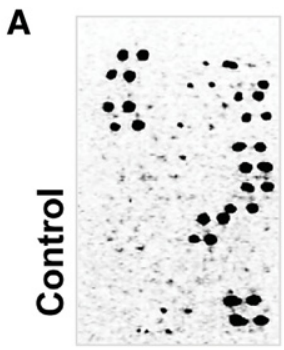

B

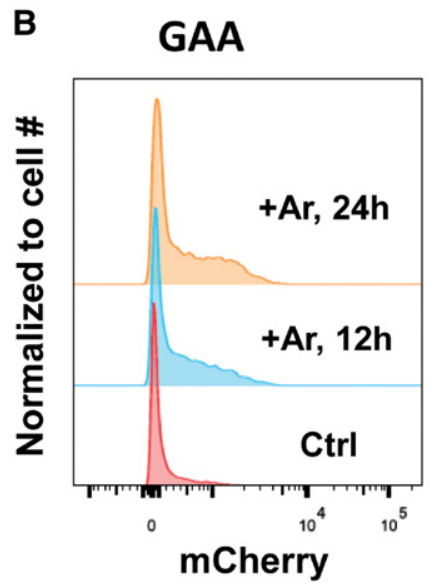

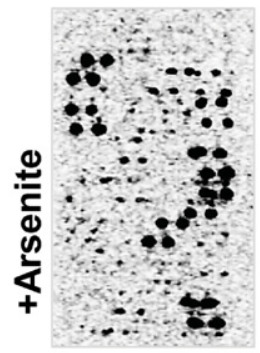

C GAG

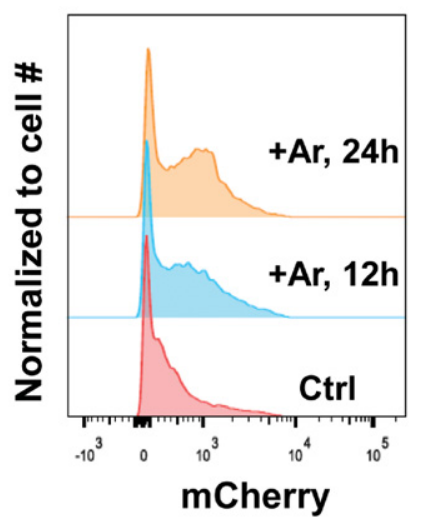

D

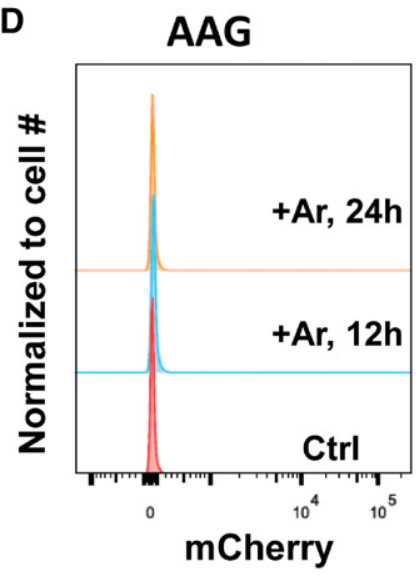

FIGURE 3. Oxidative stress increases mistranslation of GAA (Glu) and GAG (Glu) mCherry stable lines. (A) tRNA microarrays showing increase of misacylation upon arsenite treatment. Array grid shows the spots for cognate Met-tRNAs. $(B)$ Histogram shows the distribution of mCherry fluorescence intensity for GAA (Glu) cells. $(C)$ Histogram shows the distribution of mCherry fluorescence intensity for GAA (Glu) cells. (D) Histogram shows the distribution of mCherry fluorescence intensity for AAG (Lys) cells. In all cases, the histograms represent the average of at least three experimental replicates from two separate experiments. 

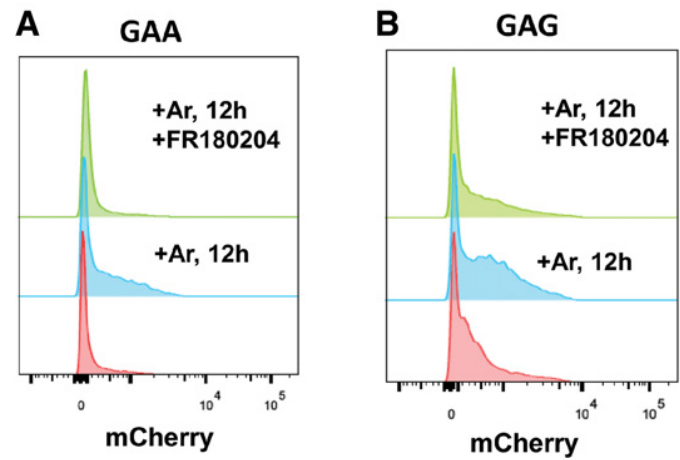

FIGURE 4. ERK-selective inhibitor decreases mistranslation in GAA (Glu) and GAG (Glu) stable lines during oxidative stress. Cells stably transfected with mCherry mutants were incubated with or without the FR180204 inhibitor and Arsenite for $12 \mathrm{~h}$, followed by flow cytometry to determine the proportion of cells with mCherry fluorescence. $(A)$ Histogram shows the distribution of mCherry fluorescence intensity for GAA (Glu) cells. (B) Histogram shows the distribution of mCherry fluorescence intensity for GAG (Glu) cells. In all cases, the histograms represent the average of at least three experimental replicates from two separate experiments.

\section{Effect of tRNA isodecoders on mistranslation}

Next, we set out to explore an application for the newly developed reporter system. Isodecoder tRNAs have the same anticodon but different body sequences. The mammalian genomes contain an unexpectedly large number of tRNA isodecoder genes (Goodenbour and Pan 2006; Chan and Lowe 2009; Parisien et al. 2013). Isodecoder tRNAs can fine-tune the efficiency of stop codon suppression (Geslain and Pan 2010) or significantly affect ribosome stalling (Ishimura et al. 2014). We therefore decided to examine whether tRNA isodecoders also show differential effects on Met mistranslation using the GAA and GAG stable cell lines.

The human genome contains a total of seven tRNA Glu gene sequences that can easily fold into a cloverleaf secondary structure, two with CUC anticodon and five with UUC anticodon (Fig. 5A; Supplemental Fig. S7a). CUC1 and CUC2 tRNAs differ by one nucleotide in the acceptor stem (A71/G71), making either an C2-A71 or a C2-G71 pair. The UUC1 tRNA has a very distinct sequence from all other tRNA ${ }^{\mathrm{Glu}}$ s. UUC2 and UUC3 tRNAs differ by one nucleotide in the acceptor stem, making either a G7-U66 or G7-C66 pair. UUC4 and UUC5 differ by three nucleotides, two switching an A-U to G-C pair in the acceptor stem and one in the variable loop. According to our DM-tRNA-seq data on HEK293T cells (Zheng et al. 2015), the four tRNA ${ }^{\text {Glu }}$ isodecoders with more than one gene copy are expressed at high levels, whereas the three tRNA ${ }^{\text {Glu }}$ isodecoders with single gene copy are expressed at very low levels (Fig. 5B).

We monitored mistranslation changes upon transfection of the transcripts of these seven tRNA ${ }^{\text {Glu }}$ s. tRNA ${ }^{\text {Glu }}$ isodecoders were synthesized in vitro with the proper $5^{\prime}$ ends generated by RNase P cleavage, then directly transfected to stable GAA and GAG cells, as done previously (Carbon et al. 1983; Buvoli et al. 2000; Kohrer et al. 2001; Geslain and Pan 2010). The mCherry level was then analyzed by flow cytometry after $36 \mathrm{~h}$. Direct transfection of tRNA transcript at the same amount was done to avoid any potential differences in RNA polymerase III transcription due to sequence differences of the isodecoders. A major drawback of direct tRNA transfection is that these tRNAs are unlikely to be modified in full which can affect both tRNA stability and translation efficiency (Phizicky and Hopper 2010). We determined the stability of these tRNA transcripts in cells using $\alpha-{ }^{32} \mathrm{P}-\mathrm{A} 76-$ labeled transcripts. After transfection, total RNA was extracted from the cells and analyzed on denaturing PAGE (Supplemental Fig. S7b). Our results show that the stability of these seven tRNA transcripts is comparable and within 1.5 -fold from each other.

The mCherry level change shows that transfecting these isodecoders has distinct effects on mistranslation, but the magnitudes of these effects are modest (Fig. 6). CUC isodecoders are supposed to read only GAG codon; the
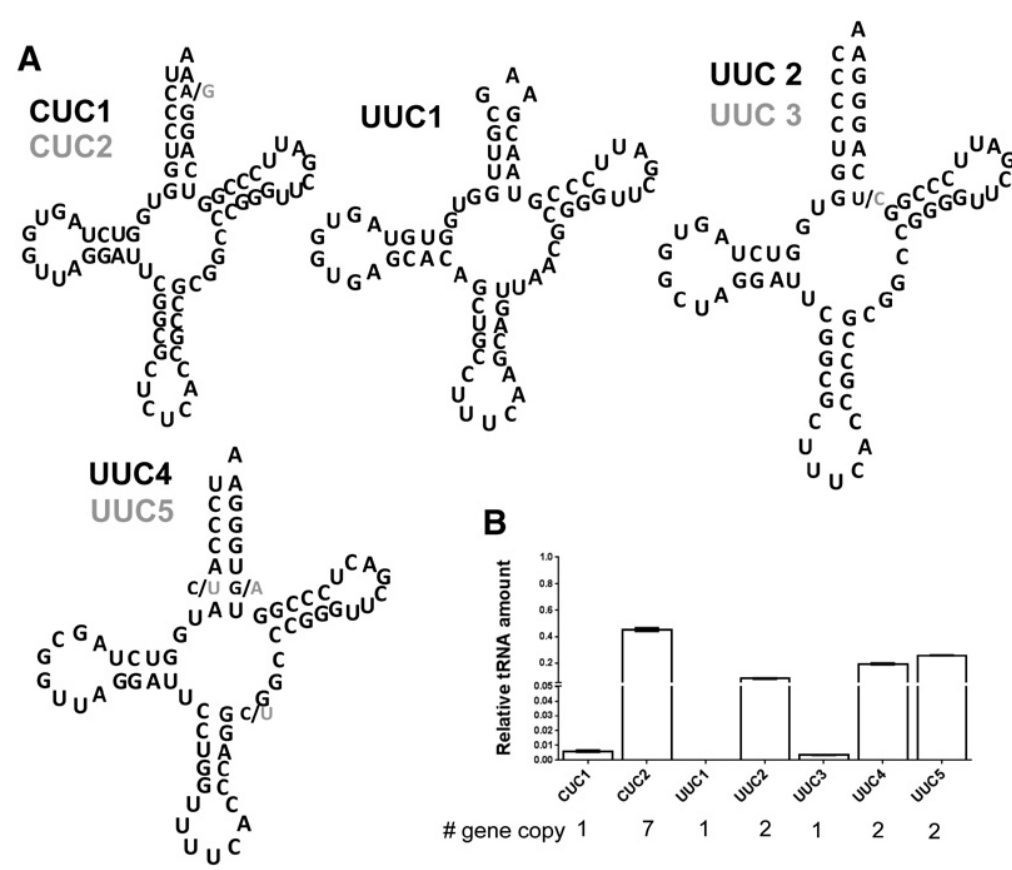

FIGURE 5. Human tRNA Glu isodecoders. (A) Secondary structure prediction according to tRNAscan-SE. Sequence swaps are indicated in red. (B) Relative tRNA ${ }^{\text {Glu }}$ quantification in HEK293T cells obtained by deep sequencing data from Zheng et al. (2015). 
transfection of CUC1 only showed an increase for GAG cells, but not for GAA cells. Transfection of CUC2, however, showed an increase in both GAG and GAA cells. UUC isodecoders are supposed to read both GAA and GAG codons; transfection of UUC1 and UUC5 showed an increase for both cells. Transfection of UUC2 and UUC4 had no effect, whereas transfection of UUC3 showed an increase for GAG cells.

Unlike mRNA transfection which often leads to overexpression of the mRNA of interest, tRNA transfection is unlikely to markedly increase the total amount of tRNA when that tRNA is already present in millions of copies in mammalian cells (Pavon-Eternod et al. 2013). In all cases, therefore, changes in the overall concentration of tRNA ${ }^{\mathrm{Glu}}$ in cells are expected to be small. However, transfection of the three tRNA $^{\text {Glu }}$ isodecoders CUC1, UUC1, and UUC3 which are present in very low amounts to begin with (Fig. 5B) can result in their overrepresentation. Transfection of these three tRNAs all show a marked increase in mistranslation, in particular for the UUC1 isodecoder. This result, however, is not unique to the "rare" isodecoders as transfection of an abundant isodecoder UUC5 also markedly increased mistranslation. These results suggest that the single copy tRNA ${ }^{\text {Glu }}$ isodecoders may be generally more active in mistranslation than the multi-copy tRNA ${ }^{\text {Glu }}$ isodecoders. Tissue-specific expression of these "rare" isodecoders may strongly affect mistranslation. On the other hand, transfected tRNAs do not go through the same tRNA maturation pathway and are likely hypomodified. Whether "rare" isodecoders are truly used more widely for mistranslation requires further investigation.

\section{CONCLUDING REMARKS}

In summary, we developed a dual-fluorescence reporter to specifically quantify mistranslation via mismethionylation of tRNA ${ }^{\text {Glu }}$ in human cells. The dual fluorescence reporter we created represents a significant advancement for the study of mistranslation in live cells. This new method allows rapid and convenient detection of misacylation under a wide range of physiological conditions, while monitoring utilization of these misacylated tRNAs for protein synthesis. The fusion of two distinct fluorescent proteins allows simultaneous quantification in the same sample and eliminates bias due to intrinsic variations in protein expression. Stable transfection of cells ensures highly reproducible, fast, and precise measurement of fluorescence emission by flow cytometry, thus potentiating the investigation of mistranslation for high-throughput analysis. We applied the new dual
A

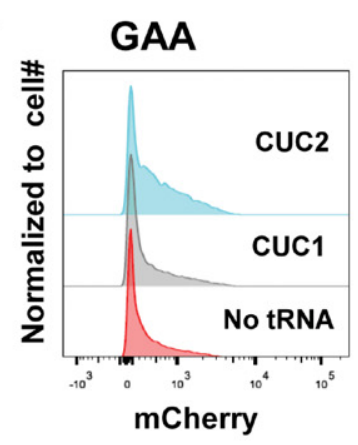

B

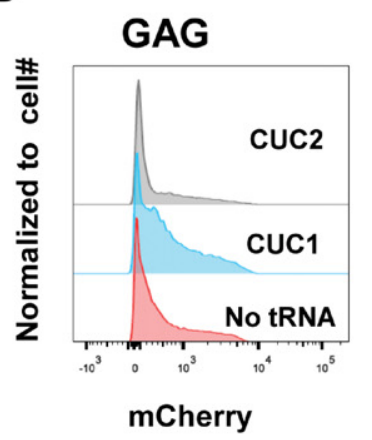

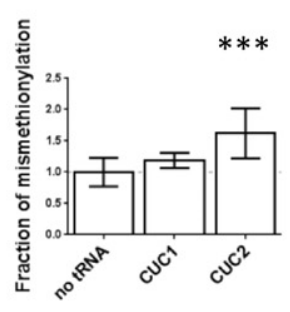
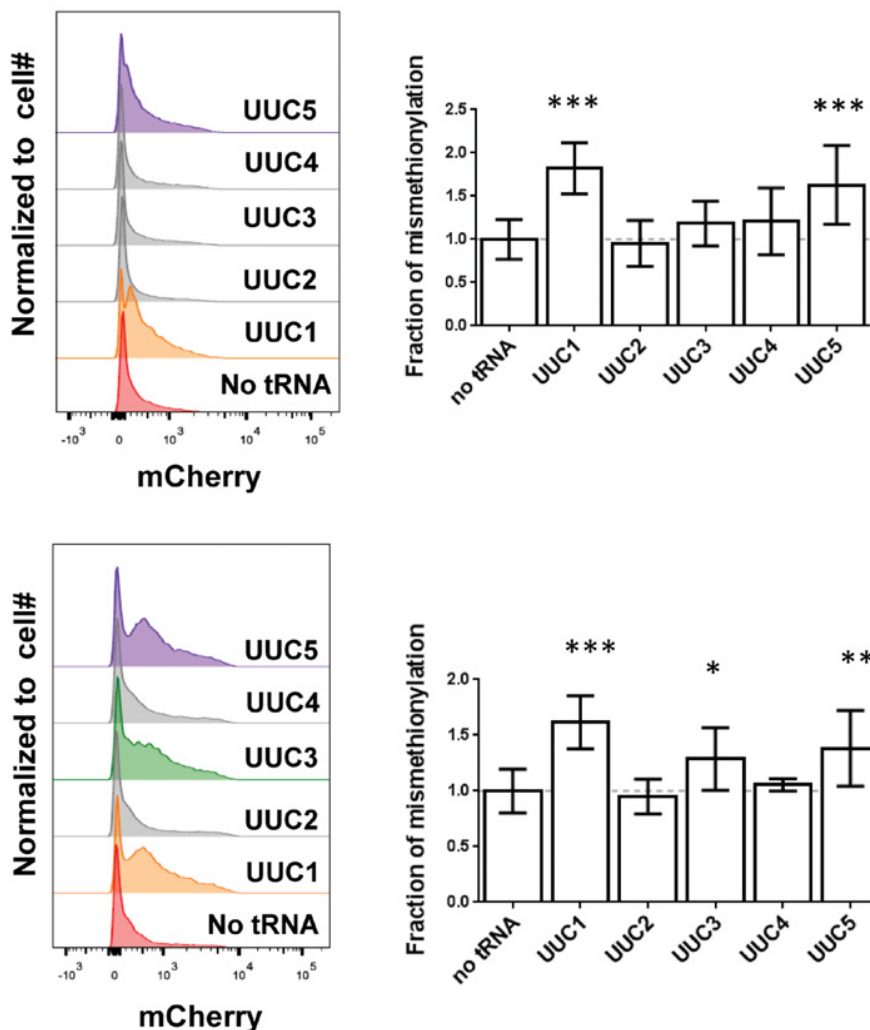

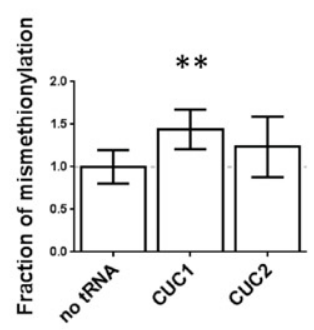

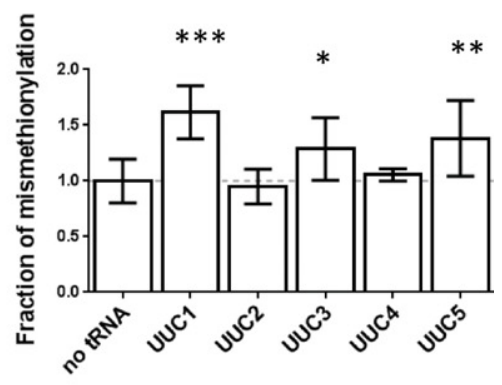

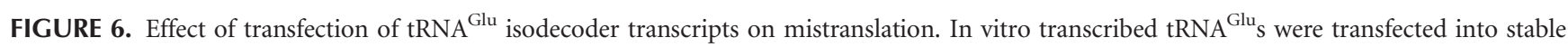
GAA/GAG mutant cells for $36 \mathrm{~h}$, followed by flow cytometry to measure mistranslation. Final values were calculated relatively to mock-transfected cells (only lipofectamine). (A) GAA stable lines. (B) GAG stable lines. Histograms represent the distribution of mCherry fluorescence intensity (average of at least three experimental replicates from two separate experiments). 
fluorescence reporter system to characterize the mistranslation efficiency of tRNA ${ }^{\text {Glu }}$ isodecoders. Our system should be useful in the discovery of new cellular components and mechanisms involved in tRNA misacylation through chemical screening or genomic RNAi libraries.

\section{MATERIALS AND METHODS}

\section{DNA vectors}

Dual fluorescence constructs containing both enhanced monomeric (A206K) EGFP (mammalian codon optimized) and mCherry were inserted into the backbone of pEGFP-C1 vector (Clontech). Mutation of Met72 to Glu and Lys in mCherry was performed by site-directed mutagenesis in the vector sequence and verified by DNA sequencing using the following primer pairs: $5^{\prime}$-CTCGCCG ACCACTACCAGCAG and 5'-CCACGATGGTGTAGTCCTCG. Purification of plasmid DNA was completed using the Qiagen Maxi Prep Kit, in accordance with the manufacturer's protocol.

\section{Cell culture}

Both HEK293 cells and stably transfected lines were cultured at $37^{\circ} \mathrm{C}$ with $5 \% \mathrm{CO}_{2}$ in Dulbecco's Modified Eagle's (DMEM) high-glucose medium supplemented with $10 \%$ heat-inactivated fetal bovine serum, L-glutamine, and $1 \%$ penicillin/streptomycin. HeLa cells were grown in DMEM with 10\% fetal bovine serum (FBS), 100 $\mathrm{U} / \mathrm{mL}$ penicillin, and $100 \mathrm{mg} / \mathrm{mL}$ streptomycin at $37^{\circ} \mathrm{C}, 5 \% \mathrm{CO}_{2}$.

\section{Plasmid transfection}

For transient transfections, cells were cultured in 96-well plates to $70 \%$ confluence and transfected with 10-60 ng of plasmid DNA per well using Lipofectamine (Invitrogen), following the manufacturer's instructions.

\section{Generation of stable cell lines}

To establish stably transfected lines, HEK293 cells were plated on 6well plates in density of $6 \times 10^{5}$ cells per well and without antibiotics. The following day, cells were transfected with $4 \mu \mathrm{g}$ of plasmid DNA using Lipofectamine2000 according to the manufacturer's instructions. Geneticin was added to the cell culture, starting with 100 $\mu \mathrm{g} / \mathrm{mL}$. Geneticin concentration was increased every $4 \mathrm{~d}$ by 100 $\mu \mathrm{g} / \mathrm{mL}$ until a final concentration of $1000 \mu \mathrm{g} / \mathrm{mL}$ was reached. The medium was changed every $2 \mathrm{~d}$ to discard dead cells. After geneticine pressure cells were sorted by flow cytometer and based on GFP fluorescence intensity, to obtain single cell cultures. Only 5\% of the cells with the strongest fluorescence were gated and used for further culture.

\section{E. coli FACS}

Escherichia coli DH10B cells harboring pQE81 (empty vector), pQE81-EGFP-mCherry, or pQE81-EGFP-mCherry(mut) were grown in Luria broth supplemented with $100 \mu \mathrm{g} / \mathrm{mL}$ carbenicilin. Cells were grown to $\mathrm{OD}_{600} \sim 0.5$ in baffled Erlenmeyer flasks at $37^{\circ} \mathrm{C}$ with shaking. Protein expression was induced by addition of
$1 \mathrm{mM}$ IPTG for $4 \mathrm{~h}$. Cytometry was carried out on a BD-LSR II flow cytometer. mCherry fluorescence was collected using PETexas red channels $(610 \pm 20 \mathrm{~nm})$.

\section{tRNA misacylation microarray}

${ }^{35} \mathrm{~S}$-Met pulse labeling of human cells and acylated tRNA extraction were performed as described previously (Netzer et al., 2009). Array hybridization was performed on a Genomic Solutions Hyb4 station in $2 \times \mathrm{SSC}, \mathrm{pH} 4.8$, at $60^{\circ} \mathrm{C}$ for $50 \mathrm{~min}$, followed by exposure to phosphorimaging.

\section{Identification of GFP-mCherry peptides using MALDI-TOF/TOF}

HEK 293T cells grown on $15 \mathrm{~cm}$ cell culture plates were transfected with $40 \mu \mathrm{g}$ GFP-mCherry plasmid (WT, GAA, or GAG) using Lipofectamine 2000 (Invitrogen) according to the manufacturer's instruction. Cells were collected after $48 \mathrm{~h}$ for immunoprecipitation with anti-GFP antibody (Abcam or Santa Cruz). Purified proteins were eluted from protein A beads (Invitrogen) using 0.1 M glycine, $\mathrm{pH} 3$, and analyzed by SDS-PAGE. $5 \mu \mathrm{g}$ of GFP-mCherry proteins were digested with $50 \mathrm{ng} / \mu \mathrm{L}$ endoproteinase Glu-C (New England Biolabs) at $37^{\circ} \mathrm{C}$ for $24 \mathrm{~h}$. Digested peptides were mixed with MALDI matrix 2,5-dihydroxybenzoic acid (Bruker) and analyzed in an MALDI-TOF/TOF mass spectrometer (Bruker).

\section{Oxidative stress induction and FR180204 inhibitor treatment}

Cells were cultured in 6-well plates at a density of $6 \times 10^{5}$ cells per well. At a 50\%-60\% confluence, cells were treated with $10 \mu \mathrm{M}$ sodium arsenite (Sigma) for 12 and $24 \mathrm{~h}$. When indicated, $25 \mu \mathrm{M}$ FR180204 (Tocris) was added to the culture $1 \mathrm{~h}$ before sodium arsenite. Cells were then pelleted by centrifugation, washed, and resuspended in PBS buffer containing 10\% FBS. Analysis by flow cytometry was made using a BD FACSCanto instrument. EGFP and mCherry were measured using the FITC (fluorescein) and PE-Texas Red channels, respectively. A minimum of 10,000 events was collected. Data were analyzed with using the FlowJo software (version X10.0.r2, Tree Star, Ashland, OR). Quadrant gates were drawn based on fluorescence emission of the WT (positive control) cell population. Debris and doublets were gated out of the analysis.

\section{In vitro transcription of tRNA ${ }^{\text {Glu }}$ isodecoders}

DNA templates containing both the T7 promoter and the tRNA gene were first synthesized by PCR of overlapping oligonucleotides. Six of the seven $\mathrm{RRA}^{\mathrm{Glu}}$ isodecoders contain a $5^{\prime} \mathrm{U} / \mathrm{C}$. For these isodecoders, transcription was done using templates with added $5^{\prime}$ precursors followed by cleavage by a bacterial RNase P holoenzyme as described in detail previously (Wiltrout et al. 2012). After synthesis by T7 RNA polymerase, tRNAs were extracted from a $10 \%$ polyacrylamide by soaking gel slices overnight at $4^{\circ} \mathrm{C}$ in a $\mathrm{pH} 7$ solution of $50 \mathrm{mM} \mathrm{KOAc}$ and $200 \mathrm{mM} \mathrm{KCl}$. After ethanol precipitation, purified tRNAs were resuspended in RNase-free $\mathrm{H}_{2} \mathrm{O}$. 


\section{Transfection of tRNA isodecoder transcripts}

Cells were cultured in 24 -well plates at a density of $2 \times 10^{5}$ cells per well. After $12 \mathrm{~h}$, cells were transfected with $250 \mathrm{ng}$ tRNA/well using Lipofectamine 3000 (Life Technologies) and according to the manufacturer's instructions. $36 \mathrm{~h}$ after transfection, cells were pelleted by centrifugation, washed, and resuspended in PBS buffer containing $10 \%$ FBS. Analysis by flow cytometry was made using a BD FACSCanto instrument. EGFP and mCherry were measured using the FITC (fluorescein) and PE-Texas Red channels, respectively. A minimum of 10,000 events was collected. FlowJo software (Tree Star, Ashland, OR) was used to gate and quantify the EGFP + /mCherry+ cell population.

\section{tRNA stability assay}

tRNA isodecoder transcripts were labeled with $\left[a-{ }^{32} \mathrm{P}\right]$ ATP in a twostep process and using the recombinant $E$. coli CCA adding enzyme (Ledoux and Uhlenbeck 2008). Briefly, 40 pmol tRNAs were first renaturated by incubation in $20 \mathrm{mM}$ Tris- $\mathrm{HCl}, \mathrm{pH} 7.5$, at $85^{\circ} \mathrm{C}$ for $2 \mathrm{~min}$ followed by the addition of $10 \mathrm{mM} \mathrm{MgCl}_{2}$ and a $5 \mathrm{~min}$ incubation at $37^{\circ} \mathrm{C}$. Finally, for the CCA labeling, tRNAs were incubated in a reaction mixture containing $20 \mathrm{mM} \mathrm{MgCl}_{2}, 50 \mathrm{mM}$ glycine, $\mathrm{pH}$ 9.0, $50 \mathrm{mM}$ pyrophosphate, $20 \mathrm{mM}$ CTP, $5.5 \mathrm{pmol}\left[\mathrm{a}^{32} \mathrm{P}\right]$ ATP, and $0.5 \mu \mathrm{g}$ CCA adding enzyme. After a $10 \mathrm{~min}$ incubation $37^{\circ}$ C, tRNAs were gel-purified and transfected to cells in 24-well plates using Lipofectamine 3000 reagent (Life Technologies) according to the manufacturer's instructions. Total RNAs were extracted with TRIzol (Ambion) after $36 \mathrm{~h}$ and loaded on $10 \%$ polyacrylamide urea gel. The gel was then exposed to a phosphorimager screen and imaged.

\section{tRNA sequencing}

DM-tRNA-seq data were from a previously published work ([Zheng et al. 2015], deposited as GSE66550). Sequencing reads were aligned using Bowtie to a modified hg19 genomic tRNA database. Briefly, a tRNA library was adapted from the tRNAScan-SE library by appending CCA to tRNAs from the genomic tRNA database (http:/gtrnadb.ucsc.edu/Hsapi19/). Isodecoders with identical scores were consolidated for ease of identity assignment, decreasing the number of reference genes and pseudogenes from 625 to 462 . Prior to mapping, reads were processed using Trimmomatic v0.32. Sequences greater than $15 \mathrm{bp}$ were then aligned to the aforementioned culled tRNA library using Bowtie2 with sensitive options. Reads mapping to multiple isodecoders due to the length of fragments were discarded.

\section{Statistical analysis}

One-way ANOVA with Dunnett's post hoc test was performed using the GraphPad Prism 6.05 software. Results were considered significant at $P<0.05$.

\section{SUPPLEMENTAL MATERIAL}

Supplemental material is available for this article.

\section{ACKNOWLEDGMENTS}

This work was supported by the National Institutes of Health (DP1GM105386 to T.P.).

Received August 26, 2015; accepted November 25, 2015.

\section{REFERENCES}

Avery SV. 2011. Molecular targets of oxidative stress. Biochem J 434: 201-210.

Buvoli M, Buvoli A, Leinwand LA. 2000. Suppression of nonsense mutations in cell culture and mice by multimerized suppressor tRNA genes. Mol Cell Biol 20: 3116-3124.

Campbell RE, Tour O, Palmer AE, Steinbach PA, Baird GS, Zacharias DA, Tsien RY. 2002. A monomeric red fluorescent protein. Proc Natl Acad Sci 99: 7877-7882.

Carbon P, Haumont E, Fournier M, de Henau S, Grosjean H. 1983. Sitedirected in vitro replacement of nucleosides in the anticodon loop of tRNA: application to the study of structural requirements for queuine insertase activity. EMBO J 2: 1093-1097.

Carter CW Jr. 1993. Cognition, mechanism, and evolutionary relationships in aminoacyl-tRNA synthetases. Annu Rev Biochem 62: 715-748.

Chan PP, Lowe TM. 2009. GtRNAdb: a database of transfer RNA genes detected in genomic sequence. Nucleic Acids Res 37: D93-D97.

Dittmar KA, Mobley EM, Radek AJ, Pan T. 2004. Exploring the regulation of tRNA distribution on the genomic scale. J Mol Biol 337: 31-47.

Dittmar KA, Goodenbour JM, Pan T. 2006. Tissue-specific differences in human transfer RNA expression. PLoS Genet 2: e221.

Geslain R, Pan T. 2010. Functional analysis of human tRNA isodecoders. J Mol Biol 396: 821-831.

Goodenbour JM, Pan T. 2006. Diversity of tRNA genes in Eukaryotes. Nucleic Acids Res 34: 6137-6146.

Ibba M, Soll D. 2000. Aminoacyl-tRNA synthesis. Annu Rev Biochem 69: 617-650.

Imlay JA. 2008. Cellular defenses against superoxide and hydrogen peroxide. Annu Rev Biochem 77: 755-776.

Ishimura R, Nagy G, Dotu I, Zhou H, Yang XL, Schimmel P, Senju S, Nishimura Y, Chuang JH, Ackerman SL. 2014. Ribosome stalling induced by mutation of a CNS-specific tRNA causes neurodegeneration. Science 345: 455-459.

Jones TE, Alexander RW, Pan T. 2011. Misacylation of specific nonmethionyl tRNAs by a bacterial methionyl-tRNA synthetase. Proc Natl Acad Sci 108: 6933-6938.

Kohrer C, Xie L, Kellerer S, Varshney U, RajBhandary UL. 2001. Import of amber and ochre suppressor tRNAs into mammalian cells: a general approach to site-specific insertion of amino acid analogues into proteins. Proc Natl Acad Sci 98: 14310-14315.

Kwon NH, Kang T, Lee JY, Kim HH, Kim HR, Hong J, Oh YS, Han JM, Ku MJ, Lee SY, et al. 2011. Dual role of methionyl-tRNA synthetase in the regulation of translation and tumor suppressor activity of aminoacyl-tRNA synthetase-interacting multifunctional protein-3. Proc Natl Acad Sci 108: 19635-19640.

Ledoux S, Uhlenbeck OC. 2008. [ $\left.33^{\prime}-{ }^{32} \mathrm{P}\right]$-labeling tRNA with nucleotidyltransferase for assaying aminoacylation and peptide bond formation. Methods 44: 74-80.

Lee JY, Kim DG, Kim BG, Yang WS, Hong J, Kang T, Oh YS, Kim KR, Han BW, Hwang B, et al. 2014. Promiscuous methionyl-tRNA synthetase mediates adaptive mistranslation against oxidative stresses. J Cell Sci 127: 4234-4245.

Luo S, Levine RL. 2009. Methionine in proteins defends against oxidative stress. FASEB J 23: 464-472.

Netzer N, Goodenbour JM, David A, Dittmar KA, Jones RB, Schneider JR, Boone D, Eves EM, Rosner MR, Gibbs JS, et al. 2009. Innate immune and chemically triggered oxidative stress modifies translational fidelity. Nature 462: 522-526.

Ohori M, Kinoshita T, Okubo M, Sato K, Yamazaki A, Arakawa H, Nishimura S, Inamura N, Nakajima H, Neya M, et al. 2005. 


\section{Gomes et al.}

Identification of a selective ERK inhibitor and structural determination of the inhibitor-ERK2 complex. Biochem Biophys Res Commun 336: 357-363.

Olejniczak M, Uhlenbeck OC. 2006. tRNA residues that have coevolved with their anticodon to ensure uniform and accurate codon recognition. Biochimie 88: 943-950.

Parisien M, Wang X, Pan T. 2013. Diversity of human tRNA genes from the 1000-genomes project. RNA Biol 10: 1853-1867.

Pavon-Eternod M, Gomes S, Rosner MR, Pan T. 2013. Overexpression of initiator methionine tRNA leads to global reprogramming of tRNA expression and increased proliferation in human epithelial cells. RNA 19: 461-466.

Phizicky EM, Hopper AK. 2010. tRNA biology charges to the front. Genes Dev 24: 1832-1860.

Sankaranarayanan R, Moras D. 2001. The fidelity of the translation of the genetic code. Acta Biochim Pol 48: 323-335.
Schimmel PR, Soll D. 1979. Aminoacyl-tRNA synthetases: general features and recognition of transfer RNAs. Annu Rev Biochem 48: 601-648.

Shaner NC, Campbell RE, Steinbach PA, Giepmans BN, Palmer AE, Tsien RY. 2004. Improved monomeric red, orange and yellow fluorescent proteins derived from Discosoma sp. red fluorescent protein. Nat Biotechnol 22: 1567-1572.

Wiltrout E, Goodenbour JM, Frechin M, Pan T. 2012. Misacylation of tRNA with methionine in Saccharomyces cerevisiae. Nucleic Acids Res 40: 10494-10506.

Wolfson AD, LaRiviere FJ, Pleiss JA, Dale T, Asahara H, Uhlenbeck OC. 2001. tRNA conformity. Cold Spring Harb Symp Quant Biol 66: 185-193.

Zheng G, Qin Y, Clark WC, Dai Q, Yi C, He C, Lambowitz AM, Pan T. 2015. Efficient and quantitative high-throughput tRNA sequencing. Nat Methods 12: 835-837. 

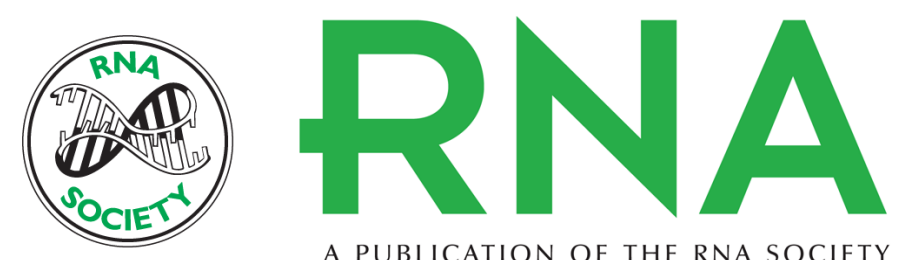

A PUBLICATION OF THE RNA SOCIETY

\section{A dual fluorescent reporter for the investigation of methionine mistranslation in live cells}

Ana Cristina Gomes, Anna J. Kordala, Rita Strack, et al.

RNA 2016 22: 467-476 originally published online January 4, 2016

Access the most recent version at doi:10.1261/rna.054163.115

\section{Supplemental http://rnajournal.cshlp.org/content/suppl/2015/12/22/rna.054163.115.DC1 Material}

References This article cites 31 articles, 11 of which can be accessed free at: http://rnajournal.cshlp.org/content/22/3/467.full.html\#ref-list-1

Creative This article is distributed exclusively by the RNA Society for the first 12 months after the Commons License full-issue publication date (see http://rnajournal.cshlp.org/site/misc/terms.xhtml). After 12 months, it is available under a Creative Commons License (Attribution-NonCommercial 4.0 International), as described at http://creativecommons.org/licenses/by-nc/4.0/.

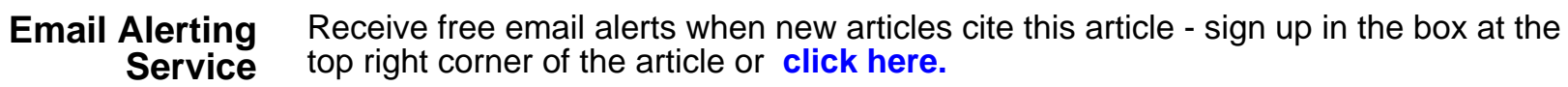

